\title{
Effect of Magnetic Field on Dilepton Production Rate in Relativistic Heavy Ion Collisions
}

\author{
P. K. Sethy ${ }^{1 *}$, Y. Kumar ${ }^{2}$, S. S. Singh ${ }^{1}$ \\ ${ }^{1}$ Department of Physics and Astrophysics, University of Delhi, Delhi, India \\ ${ }^{2}$ Department of Physics, Deshbandhu College, Kalkaji, New Delhi, India \\ Received 23 October 2019, accepted in final revised form 3 January 2020
}

\begin{abstract}
It is believed that a transient strong magnetic field is generated in heavy-ion collision. The strength of this field perpendicular to the reaction plane and is estimated to be around $\mathrm{eB}=0.03 \mathrm{GeV}^{2}$ at RHIC and $\mathrm{eB}=0.3 \mathrm{GeV}^{2}$ at $\mathrm{LHC}$. We study the effect of this magnetic field on dilepton yield using a modified quasi particle model. The results show a clear enhancement in dilepton yield and our result is in good agreement with the recently reported results.
\end{abstract}

Keywords: Quark-Gluon Plasma; QCD; Dilepton; Heavy-ion collision.

๑) 2020 JSR Publications. ISSN: 2070-0237 (Print); 2070-0245 (Online). All rights reserved. doi: http://dx.doi.org/10.3329/jsr.v12i2.43938 J. Sci. Res. 12 (2), 215-221 (2020)

\section{Introduction}

The contradiction between the limiting temperature concept of hadronic matter from Hegedron model and monotonically increase of energy density with collision energy concept from Fermi-Landau-Pomeranchuk model, indicates existence of a deconfined quark phase. Relativistic heavy-ion collision experiments have confirmed the presence of strongly coupled matter- Quark-Gluon Plasma (QGP) [1-3]. Due to considerable interaction strength this system of liberated quarks and gluons reaches its thermalized state early. This thermalized quark matter expands and gradually cools down as the system evolves with time and beyond a critical temperature it converts into hadronic matter. The hadron gas further expands to reduce energy density and finally reaches the freeze-out.

The contemporary physics provides a theoretical platform to understand the manybody physics of Quantum Chromodynamics (QCD). QCD matter at low temperature and density is composed of hadrons, while at high temperature and high density; it is

\footnotetext{
*orresponding author: psethy@kmc.du.ac.in
} 
believed that the deconfinement of quarks and gluons takes place. The wealth of information provided by the Relativistic Heavy Ion Collider (RHIC) at Brookhaven National Laboratory (BNL) and by the Large Hadron Collider (LHC) at CERN has confirmed the formation of such a QCD matter-hot quark-gluon plasma (QGP). The off-central heavy-ion collision produce large electromagnetic fields [4,5]. Strong magnetic field has also been estimated in noncentral heavy-ion collisions [6-9]. When two heavy ion nuclei approach towards each other generate two electric currents in opposite directions and eventually produce a large time dependent magnetic field perpendicular to the collision plane. Using Biot-Savart formulae, a naive estimation of the magnetic field can be made. In RHIC, $\mathrm{Au}+\mathrm{Au}$ collisions at $\mathrm{s}=\sqrt{2} 200 \mathrm{GeV}$ has reported the magnetic field produced is in the order of $10^{19}$ Gauss and in $\mathrm{LHC} \mathrm{Pb}+\mathrm{Pb}$ collisions at $\mathrm{s}=\sqrt{ } 2.76 \mathrm{TeV}$ the magnetic field is of the order of $10^{20}$ Gauss [10-12]. Thus the experimental results reported by PHENIX at RHIC $(\mathrm{E} \leq 200 \mathrm{GeV})$ established the fact that a transient strong magnetic field is created in heavy-ion collision. This magnetic field is much larger than the mass squared of light quarks and thus are expected to affect the dynamics of the quark-gluon plasma significantly. This field is found to be much above Schwinger critical value. In this regime the classical electrodynamics breaks down. Due to high temperature and density, the direct access of QGP properties is not very feasible. Only information is available from the final state particle spectra. Therefore, it is imperative to study the magnetic field induced dilepton production in heavy-ion collision. The magnitude of the magnetic field produced is found to be linearly dependent on the collision energy [13-15] and this is evident from Lorentz gamma factor appearing in Biot-Savart formulae:

$$
e B=\frac{\gamma \alpha Z}{R_{A}^{2}}
$$

A relativistic electron carries with it a virtual photon spectrum of photons per unit frequency interval. When radiation occurs, for whatever reason, the observed frequency spectrum will closely follow this virtual spectrum. In cases where the driving force for the radiation extends over many formation lengths, the spectrum of radiated photons per unit path length for intense processes which describe the radiation emitted over one formation length, divided by the formation length [16]. This is referred to as Weizsacker-William's approximation and is useful to compute the dilepton yield with logarithmic accuracy. Initially just after the collision of heavy ions, the initial distribution of soft $(\mathrm{E}<\mathrm{T})$ and hard quarks $\left(\mathrm{E}>\mathrm{T}\right.$ and $\left.\mathrm{Q}_{\mathrm{s}}>\mathrm{T}\right)$ would be a decisive factor for the subsequent stage of emission of lepton pair. The dilepton yield is calculated for $\mathrm{T}<\mathrm{E}<\mathrm{Q}_{\mathrm{s}}$. The reason for considering this zone is that the soft quark spectrum falls off in an exponential manner whereas the hard quark spectrum is logarithmic. The numbers of soft quarks are proportional to the $\mathrm{T}^{3}$ and volume of the plasma. The number of hard quarks is proportional to square of the saturation momenta. At early times, the volume of the plasma is also proportional to the crosssectional area of heavy ion overlap region. The magnetic field in quark-gluon plasma appears to be extremely strong and slowly varying function of time for most of the 
plasma lifetime. At RHIC it decreases from $\mathrm{eB}\left(=2.5 \mathrm{GeV}^{2}\right)$ right after the collision to $\mathrm{eB}\left(=0.25 \mathrm{GeV}^{2}\right)$. This has a profound impact on all the processes occurring in QGP. The exotic state of matter is created along with a huge magnetic field. Thus, it offers a great opportunity to observe the interaction of this magnetic field with the quark-gluon plasma. Here we try to calculate the dilepton production rate with reference to the response of the QGP to the electromagnetic field.

In this paper we compute the dilepton production rate from the quark-gluon plasma in the presence of magnetic field. We envisage the situation from a different perspective. Firstly, we consider the exotic state of matter as a system of quasi particles. The quarks acquire a dynamic mass in this system due to interaction. This quark mass is considered to be temperature dependent. It has been well established that there is strong magnetic field generated at the early time of the heavy ion collisions. This magnetic field would affect the dynamics of QGP. Therefore, it is pertinent to study the effect of this magnetic field on the dilepton yield.

The structure of our paper is as follows. In section 2 we calculate production rate of dileptons at finite temperature $T$ and quark chemical potential, $\mu$ in the inherent magnetic field and brief model description. In Section 3 we present our results and compare them with some other theoretical results.

\section{Calculation of Dilepton Emission Rate and Model Description}

In heavy-ion collision, dileptons are produced from quarks (or antiquarks) through the mediation of a virtual photon. Here we consider a process where both the quark and the lepton move in the magnetic field created during the heavy-ion collision. Although there may be another possibility in which either the quark or the lepton moves in the magnetic field [17]. But it is believed that the earlier one (both quark and lepton move) is most sensitive to the prevailing magnetic field in heavy-ion collision [18].

At RHIC and LHC, the centre-of-mass energy per nucleon is larger than the ratio of nucleon mass to the product of nuclear radius and electrical conductivity. This condition is based on a reasonable approximation that the time dependence of the magnetic field is adiabatic [19]. The relativistic electron has the electromagnetic field which is very similar to the fields of a plane wave. Therefore, we can consider the relativistic electron as if a cloud of virtual photons is carried by it and has the ability to radiate. Weiszacker and Williams estimated the energy response and the number of photons corresponding to a particular frequency. Essentially the observed frequency spectrum in a radiation process keeps on following the spectrum of virtual photons carried by the relativistic electron. There are several approaches to understand the interaction of charged particles. It is quite easy to describe such interaction when the speed of the particles is nearly the speed of light. In relativistic domain, one observes the contraction of electric $\mathrm{E}$ and magnetic $\mathrm{B}$ fields of such a particle into the plane that is perpendicular to the direction of motion. The magnitude of $\mathrm{E}$ and $\mathrm{B}$ fields are nearly the same and are perpendicular to one another. For an observer at rest, at certain 
distance away from the passing particle, the effects of these fields appear to be indistinguishable from those of a passing EM wave. In such circumstances, approximation of the particles EM fields as EM plane waves helps us to analyze the interaction of two ultra-relativistic (UR) charged particles. Thus, the non-central collision of two UR particles can be equivalently treated as the interaction between an UR particle and a passing EM wave [20]. Weiszacker method is useful to write down the flux of the photons emitted by a fast quark. Withe above approximations, the dilepton production rate can be written as:

$\frac{d N_{q} \rightarrow l_{1} l_{2}}{d t d \Omega d E}=\int n(\omega) \frac{d N_{\gamma} \rightarrow l_{1} l_{2}}{d t d \Omega d E} d \omega$

where $n(\omega)$ is the flux of equivalent real photon replacing virtual photon. The expression for $n(\omega)$ is given by,

$$
n(\omega)=\frac{2 Z_{q}^{2} \alpha}{\pi \omega} \ln \frac{E}{\omega\left[1+\frac{\chi E}{\omega}\right]^{1 / 3}}
$$

It has been realised that in the relativistic heavy- ion collisions, there is a significant contribution of finite baryon chemical potential in central collision. This finite baryon chemical potential $\mu$ is reported to be at energies around $\sqrt{s}_{\mathrm{s}} \leq 200 \mathrm{AGeV}$. It is further reported by the microscopic models $[21,22]$ that the colliding heavy-ions may not be fully transparent. The significance of chemical potential produced at RHIC energy has been reported by Hammon et al. [23]. It is believed that there exists non-zero chemical potential in the early stage of the plasma. This information prompted Dumitru et al. to express dilepton emission rate as a function of temperature and quark chemical potential $\mu$ of the QGP [24]. We define quark mass which depends on temperature and chemical potential and it is expressed as,

$$
m_{q}^{2}(T, \mu)=\frac{N_{c}^{2}-1}{16 N_{c}}\left[T^{2}+\frac{\mu^{2}}{\pi^{2}}\right] g^{2}
$$

where $\mathrm{g}^{2}=4 \pi \alpha_{\mathrm{s}}$ is the QCD coupling factor and the value of $\alpha_{\mathrm{s}}$ is given as,

$$
\alpha_{s}=\frac{4}{\left(33-2 N_{f}\right) \ln \left(1+\frac{k^{2}}{\Lambda^{2}}\right)}
$$

Here $\Lambda$ is QCD parameter, $\mathrm{N}_{\mathrm{f}}$ is the number of quark flavour and $\mathrm{k}$ is the momentum value. When the plasma is exposed to magnetic field, the quark mass is suitably modified to $\mathrm{m}^{\mathrm{B}}$ eff - Magnetized Effective Quark Mass (MEQM). The expression for the magnetized effective quark mass is [25]:

$$
m_{e f f}^{B}=m_{e f f}^{2}+e B(2 n+s+1)
$$

Where $m_{\text {eff }}$ is given by Ref. [26,27],

$$
m_{e f f}^{2}=m_{c}^{2}+\sqrt{2} m_{c} m_{q}+m_{q}^{2}
$$


The parameter $\mathrm{m}_{\mathrm{c}}$ and $\mathrm{m}_{\mathrm{q}}$ are the current and thermal mass of the quark. The estimated magnetic field can be expressed as:

$$
e B=\frac{\alpha z R \sigma}{t^{2}} \exp \left(-\frac{R^{2} \sigma}{4 t}\right)
$$

In such a scenario, the single particle energy eigen value is given by [28,29],

$$
E^{B}=\left[m_{e f f}^{B}+k^{2}\right]^{1 / 2}
$$

Realizing the dependence of dilepton yield on rapidity, collision energy, saturation momentum, valence quark distribution function and some phenomenological parameter, one can simplify the expression for dilepton production rate as [30]:

$$
\frac{d N_{q \rightarrow l_{1} l_{2}}}{d t d \Omega d E}=\frac{N S Q^{2} \alpha^{2} m_{l}^{2}}{3 \pi^{5}} \sum_{i=0} Z_{q}^{2} \int_{0}^{\pi / 2} d \beta \int_{0}^{Y / 2} d y f(y) \int_{E}^{\omega_{n /}} d \omega \ln \frac{E}{\omega\left[1+(\chi E / \omega)^{1 / 3}\right]}\left[\frac{2}{x}-\chi x^{1 / 2}\right] A i(x)
$$

\section{Results}

Magnetic field generated in heavy-ion collisions has a profound impact on dilepton production. Indeed, magnetic field strength by far exceeds the critical Schwingers value during the entire QGP lifetime. The effective quark mass in the presence of magnetic field $\left(\mathrm{B}=0.03-0.3 \mathrm{GeV}^{2}\right)$ shows very similar result for dilepton yield with the recent result of Tuchin et al. Our model result also shows an enhanced dilepton yield in low invariant mass region as comparison to high mass region. Fig. 1 depicts the dilepton yield with the effective quark mass at different energy with the inclusion of magnetic field and we try to show a comparative dilepton yield. In this it is pertinent that there is an enhanced value. We attribute this enhanced value to the magnetized effective quark mass.

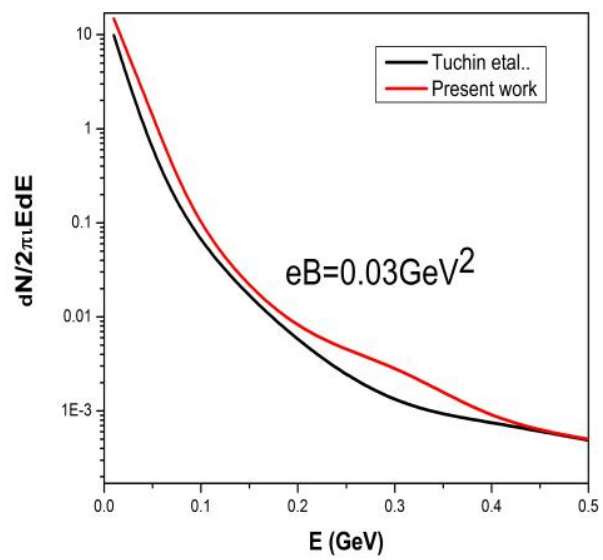

Fig. 1. Comparative dilepton yield with respect to energy at $\mathrm{eB}=0.03 \mathrm{GeV}^{2}$. 


\section{Acknowledgment}

We are very thankful to A. N. Tawfik for the constructive suggestions. The author S. S. Singh would like to thank the University of Delhi for providing the research and development strengthen fund to pursue this work.

\section{References}

1. K. Adcox et al., Nucl. Phys. A 757, 184 (2005). https://doi.org/10.1016/j.nuclphysa.2005.03.086

2. B. B. Back et al., Nucl. Phys. A 757, 28 (2005). https://doi.org/10.1016/j.nuclphysa.2005.03.084

3. K. Aamodt et al., Phys. Rev. Lett. 105, 252301 (2010). https://doi.org/10.1103/PhysRevLett.105.252301

4. V. Skokov, A. Y.Illarionov, and V. Toneev, Int. J. Mod. Phys. A 24, 5925 (2009). https://doi.org/10.1142/S0217751X09047570

5. Y. Zhong, C. B. Yang, X. Cai, and S. Q. Feng, Adv. High E. Phys. 2014, 193039 (2014). https://doi.org/10.1155/2014/193039

6. S. Schramm, B. Muller, and A. J. Schramm, Mod. Phys. Lett. A 7, 973 (1992). https://doi.org/10.1142/S0217732392000860

7. D. N. Voskresensky and N. Yu. Anisimov, Sov. Phys. JETP 51, 13 (1980).

8. J. Rafelski and B. Muller, Phys. Rev. Lett. 36, 517 (1976). https://doi.org/10.1103/PhysRevLett.36.517

9. S. Schramm, B. Muller, and A. J. Schramm, Phys. Lett. A 164, 28 (1992). https://doi.org/10.1016/0375-9601(92)90900-7

10. D. E. Kharzeev, L. D. McLerran, and H. J. Warringa, Nucl. Phys. A 803, 227 (2008). https://doi.org/10.1016/j.nuclphysa.2008.02.298

11. J. Bloczynski, X. G. Huang, X. Zhang, and J. Liao, Nucl. Phys. A 939, 85 (2015). https://doi.org/10.1016/j.nuclphysa.2015.03.012

12. Y. Zhong, C. B. Yang, X. Cai, and S. Q. Feng, Chin. Phys. C 39, 104105 (2015). https://doi.org/10.1088/1674-1137/39/10/104105

13. J. Alam, S. Raha, and B. Sinha, Phys. Rep. 273, 243 (1996). https://doi.org/10.1016/0370$\underline{1573(95) 00084-4}$

14. A. Bzdak and V. Skokov, Phys. Lett. B 710, 171 (2012). https://doi.org/10.1016/i.physletb.2012.02.065

15. K. Tuchin, Phys. Rev. C 88, 024911 (2013). https://doi.org/10.1103/PhysRevC.88.024910

16. M. S. Zolotorev, K. T. McDonald-arXiv preprint physics/0003096 (2000).

17. K. Tuchin, Phys. Rev. C 88, 024911 (2013). https://doi.org/10.1103/PhysRevC.88.024910

18. B. P. Nigam and M. K. Sundaresan, Can. J. Phys. 65, 421 (1987). https://doi.org/10.1139/p87-053

19. S. C. Ahern and John W. Norbury Phys. Rev. D 68, 113001 (2003). https://doi.org/10.1103/PhysRevD.68.113001

20. G. Gustafson - Proc. of the Workshop on Relativistic Heavy-Ion Physics at Present and Future Accelerators (Budapest, 1992).

21. H. J. Mohring and J. Ranft, Z. Phys. C 52, 643 (1991). https://doi.org/10.1007/BF01562339

22. N. Hammon, H. Stöcker, and W. Greiner, Phys. Rev. C 61, 014901 (1999). https://doi.org/10.1103/PhysRevC.61.014901

23. A. Dumitru, D. H. Rischke, T. Schonfeld, L. Winckel-omann, H. Stocker, and W. Greiner, Phys. Rev. Lett. 70, 2860 (1993). https://doi.org/10.1103/PhysRevLett.70.2860

24. Y. Kumar and S. S. Singh, Can. J. Phys. 90, 950 (2012). https://doi.org/10.1139/p2012$\underline{089}$ 
25. Y. Kumar, JPS Conf. Proc. 26, 024028 (2019).

26. P. K. Srivastava, S. K. Tiwari, and C. P. Singh, Phys. Rev. D 82, 014023 (2010). https://doi.org/10.1103/PhysRevD.82.014023

27. Y. Kumar, EPJ Web of Conf. 182, 02070 (2018). https://doi.org/10.1051/epjconf/201818202070

28. G. Endrodi, J. High E. Phys. 04, 023 (2013). https://doi.org/10.1088/14757516/2013/04/023

29. G. Endrodi, J. High E. Phys. 07, 173 (2015). https://doi.org/10.1007/JHEP07(2015)173

30. K. Tuchin, Phys. Rev. C 88, 024910 (2013). https://doi.org/10.1103/PhysRevC.88.024910 